\title{
HCPCS Level II
}

National Cancer Institute

\section{Source}

National Cancer Institute. HCPCS LevellI. NCI Thesaurus. Code C155826.

Codes used to report products, supplies, and services not described by CPT-4 codes. 